\title{
A transmit/reflect switchable frequency selective surface based on all dielectric metamaterials
}

\author{
Fei Yu*, Jun Wang*, Jiafu Wang*, Hua Ma*, Hongliang Du*, \\ $\mathrm{Xu} \mathrm{Zhuo}^{\dagger}$ and Shaobo $\mathrm{Qu}^{*}, \S^{\prime}$ \\ *Department of Mathematics and Physics \\ College of Science, Air Force Engineering University \\ Xi'an 710051, P. R. China \\ ${ }^{\dagger}$ Electronic Materials Research Laboratory \\ Xi'an Jiaotong University, Xi'an 710049, P. R. China \\ taj23simba@foxmail.com \\ §qushaobo@mail.xjtu.edu.cn
}

\begin{abstract}
Received 8 September 2015; Revised 12 November 2015; Accepted 15 November 2015; Published 28 December 2015
In this paper, we propose a novel transmit/reflect switchable frequency selective surface (FSS) in millimeter wave band based on the effective medium theory under quasi-static limit, which is designed with square-hole elements cut from continuum dielectric plates. The building elements of the surface are composed of all dielectric metamaterial rather than metal material. With proper structural design and parameters tuning, the resonance frequencies can be tuned appropriately. The frequency response of the surface can be switched from that of a reflecting structure to a transmitting one by rotating the surface $90^{\circ}$, which means under different incident polarizations. The reflective response can be realized due to the effect of electric and magnetic resonances. Theoretical analysis shows that the reflective response arises from impedance mismatching by electric and magnetic resonances. And the transmitting response is the left-handed passband, arises from the coupling of the electric and magnetic resonances. In addition, effective electromagnetic parameters and the dynamic induced field distributions are analyzed to explain the mechanism of the responses. The method can also be used to design switchable all-dielectric FSS with continuum structures in other frequencies.
\end{abstract}

Keywords: Switchable; FSS; dielectric resonance.

\section{Introduction}

Frequency selective surface (FSS) is composed of scatter arrays arranged periodically in a planar or curved surface, which can exhibit one or more pass bands or stop bands as a kind of spatial filter. Conventionally, FSS is usually constructed from patches, slits or arbitrary geometries structures within a metallic screen arranged periodically. In the past few decades, FSS has been extensively used in a variety of microwave applications, ranging from filters, antenna reflectors, radio frequency absorbers, radomes of aircraft to wireless securities, electromagnetic shielding applications. ${ }^{1-4}$

With the rapid development of wireless and satellite communication in recent years, the demands for the capabilities of multi-function and multi-frequency systems are increasing. Thus, the dual offset antenna and dual polarized antenna have drawn more attention. The blocking interference between different antennas becomes increasingly important. Many design methods and structures are adopted to obtain the bandstop or bandpass response in FSSs manufacture. Kiani et al. had presented resistive cross dipole and conducting cross dipole structures for the building of absorb/transmit for WLAN signals. ${ }^{5}$ Bossard et al. proposed reconfigurable FSS using genetic algorithms to achieve switch bandstop response in Ku band. ${ }^{6}$ Chang et al. developed an active square loop structure as the cell element to achieve electronically switched reflecting performance. ${ }^{7}$ For the traditional FSSs mentioned above, the metallic elements are indispensable. Nevertheless, the metallic materials are problematic, especially in high power and high temperature using, and the device using metallic elements is easily detected. Therefore, it is necessary to develop the all dielectric FSSs. Because of the low absorption loss compared with metallic structures, all the dielectric FSSs have been studied extensively these years, especially at infrared frequency or even higher frequencies. ${ }^{8-10}$ At the microwave frequency range, the all dielectric FSSs are customarily designed with bandstop response. ${ }^{1-15}$ In recent years, Barton adopted guided-mode resonance method to design all-dielectric FSSs for high-power microwave applications. ${ }^{16,17}$

In this paper, we present the design of all-dielectric switchable FSSs based on the effective medium theory under quasi-static limit, ${ }^{18-20}$ which can overcome the Ohmic loss and low breakdown voltage problem under high power. For

This is an Open Access article published by World Scientific Publishing Company. It is distributed under the terms of the Creative Commons Attribution 4.0 (CC-BY) License. Further distribution of this work is permitted, provided the original work is properly cited. 


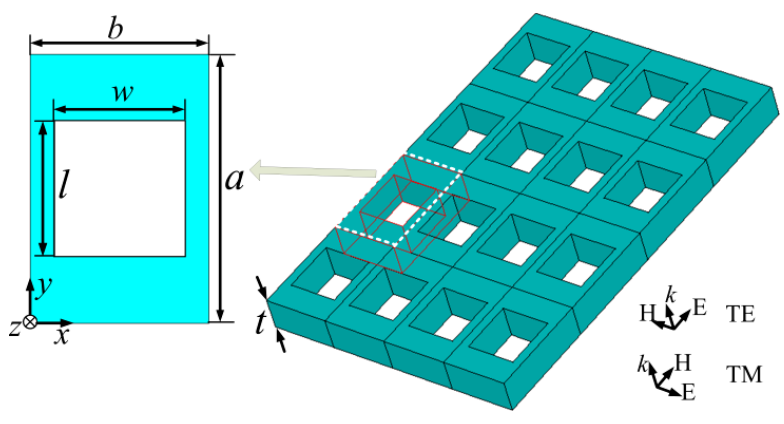

Fig. 1. Geometry of the proposed FSS and its unit cell. The geometry parameters are: $a=3.02, b=4.56, w=2.39, l=2.53, t=$ 1.47 (unit: $\mathrm{mm}$ ).

subwavelength dielectric resonators in the air background, the macroscopic electromagnetic parameters can be described by effective electromagnetic parameters. We can choose suitable unit elements and adjust the geometry parameters with required effective permittivity/permeability in framework of effective medium theory. The dynamic induced electric and magnetic distributions are investigated as an illustration. To verify this design method, a transmit/reflect switchable FSS based on all dielectric metamaterials is proposed. The switchable FSS shows a bandstop response with a sharp rolloff characteristic under transverse electric (TE) polarization, and passband response under transverse magnetic (TM) polarization. This design is implemented by cascading a twodimensional periodic array of dielectric inclusions based on the method of metamaterial design. The unit cell is shown in Fig. 1, the square-hole elements cut periodically from the continuum dielectric thin board. The appropriate and stable electric resonance (or magnetic resonance) can be achieved by adjusting the size of the structure and square-hole.

\section{Description of Structure and Principles of Operation}

The configuration of the proposed switchable all dielectric FSS is depicted schematically in Fig. 1. The structure consists of single dielectric layer, which is made of $\mathrm{Al}_{2} \mathrm{O}_{3}$ ceramics with a relative permittivity $\varepsilon_{r}=9.7$, and a loss tangent tan $\delta=0.003$. Square-hole structures are cut up periodically from the dielectric layer to obtain the unit cell of the structure, as shown in Fig. 1. The unit cell is extended periodically in $X$ and $Y$ directions. All the design parameters and all the optimized parameters are presented in Fig. 1.

By means of numerical simulation, scattering parameters can be obtained. The transmission coefficients of the proposed FSS under normal incident waves of TE polarization and TM polarization were simulated using CST Microwave Studio. Under TE polarization as shown in Fig. 2, the reflect window with the center frequency of $42 \mathrm{GHz}$. The $-10 \mathrm{~dB}$ bandwidth (BW) of the stopband is $4.15 \mathrm{GHz}$ (40.45$44.60 \mathrm{GHz})$, and the corresponding fractional bandwidth (FBW) is $9.8 \%$. The FBW can be calculated by the formula

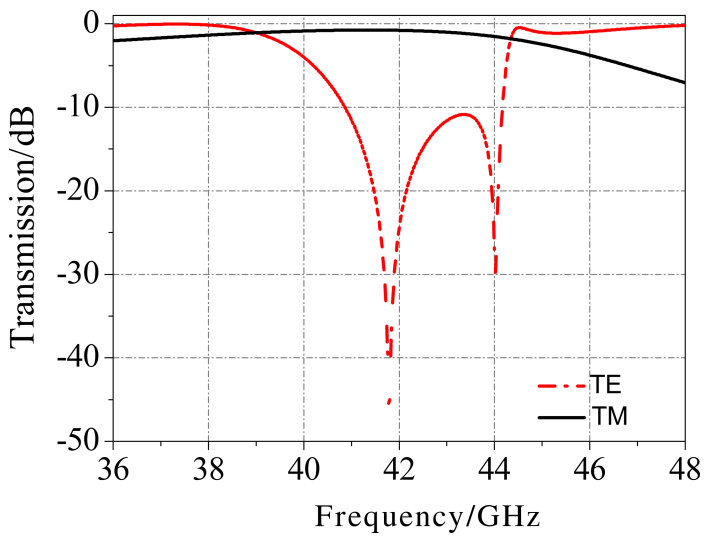

Fig. 2. Calculated frequency response of reflect/transmit switchable FSS under normally incident for TE polarization and TM polarization.

$\mathrm{FBW}=\Delta f / f_{c}=2\left(f_{2}-f_{1}\right) / f_{c}$, where $f_{c}$ is the center frequency of the stopband. But under TM polarization, the FSS gives a transmission coefficient of $-3 \mathrm{~dB}$ at the frequency range of the reflective response under TE polarization. Hence it can be made either reflective or transmitting response, according to the surface locating direction.

The effective electromagnetic parameters can be retrieved by using the scattering parameters obtained by simulation. $^{21-24}$ Figures 3(b)-3(d) show the impedance, retrieved effective permeability and permittivity of the proposed FSS, respectively under TE polarization. Figure 3(b) gives the impedance of the switchable FSS relative to the free space. It can be seen that the relative impedance of the switchable FSS in frequencies of the stopband approach to 0 , which means the impedance of the switchable FSS is mismatched to the free space. According to the law of energy conservation, the relationship between reflectivity $\rho$ and transmissivity $\tau$ is $\rho+\tau=1$, regardless of absorbing. The reflectivity $\rho$ is determined by the reflection coefficient $\Gamma$, which can be calculated by the formula

$$
\Gamma=\frac{Z-1}{Z+1} .
$$

In Eq. (1), $Z$ represents the normalized impedance calculated by

$$
Z=\frac{Z_{r}}{Z_{0}}=\sqrt{\frac{\mu_{r}}{\varepsilon_{r}}} / \sqrt{\frac{\mu_{0}}{\varepsilon_{0}}} .
$$

In Eq. (2), $Z_{r}$ represents the effective impedance of the FSS, determined by the effective electromagnetic parameters. $Z_{0}$ represents the impedance of air (approximately $377 \Omega$ ). If a bandstop response is desired, the reflectivity $\rho$ should be close to 1, which means the normalized impedance of the FSS should be 0 exactly or nearby. Therefore, in the frequency range, that is, in the frequency $40.45-44.60 \mathrm{GHz}$, the real part of the normalized impedance $Z$ approaches to 0 , leading to the stopband. ${ }^{25}$

The effective electromagnetic parameters retrieved from the calculated S parameters, as shown in Figs. 3(c) and 3(d). 


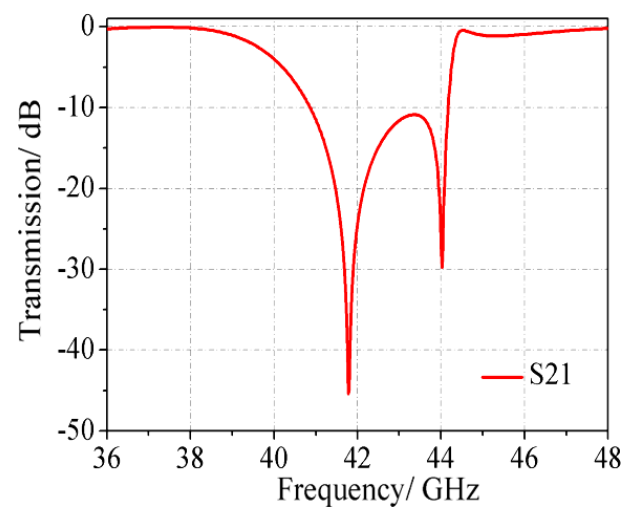

(a)

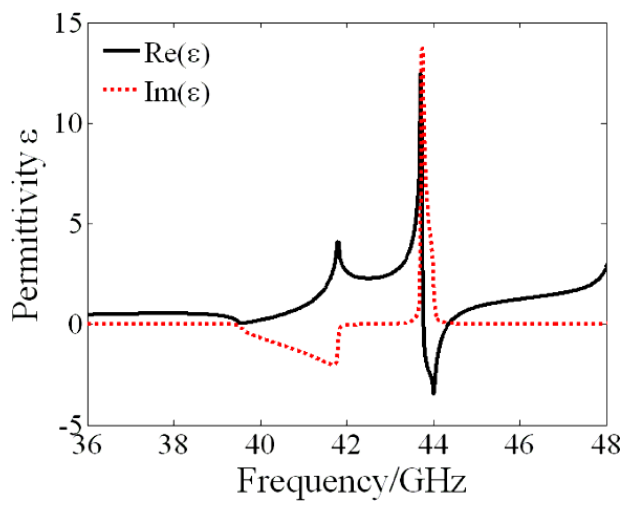

(c)

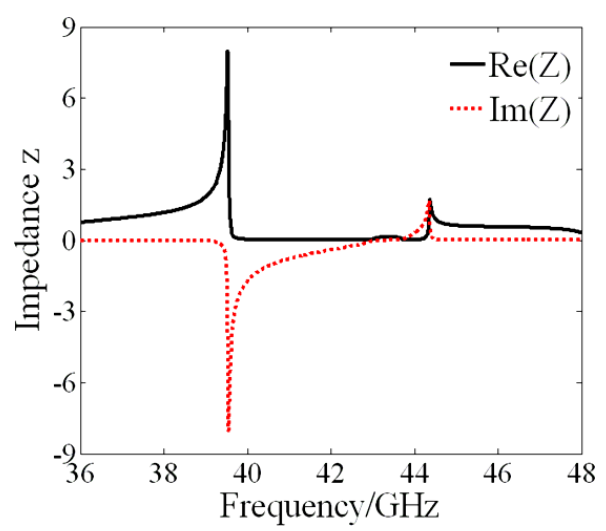

(b)

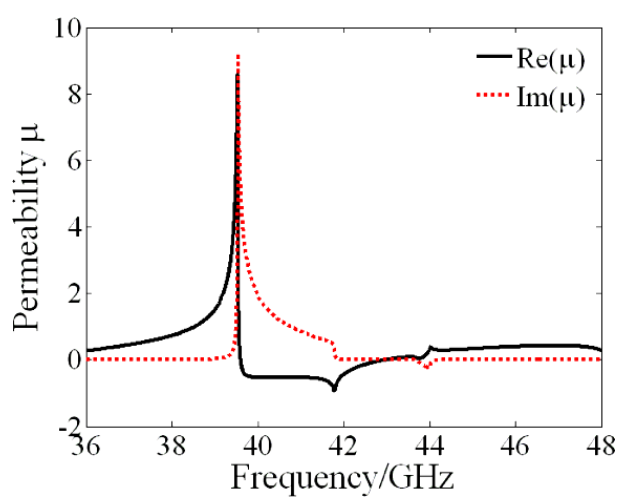

(d)

Fig. 3. Calculated frequency response of the reflect/transmit switchable FSS band under normally incident for TE polarization and the retrieved parameters: (a) transmission coefficient; (b) relative impedance; (c) relative permeability; (d) relative permittivity.

Strong electric and magnetic resonances are excited around the frequency where the effective impedance approaches to 0 . The permittivity achieves maximum first, and then falls down to the minimum with increasing frequency around $44.20 \mathrm{GHz}$. The characteristic of the permittivity curve is typical of the electric resonance, as shown in Fig. 3(c). And with increasing frequency around $39.80 \mathrm{GHz}$, the permeability also achieves maximum first, and then falls down to the minimum, which displays the magnetic resonance, as shown in Fig. 3(d). The negative parts of the effective electromagnetic parameters do not coexist in the same frequency range, and the magnetic resonance around $39.80 \mathrm{GHz}$ and the electric resonance around $44.20 \mathrm{GHz}$ result in impedance mismatching in the stopband.

The FSS can be seen as continuum dielectric resonators which possess different resonant modes to modulate the electromagnetic response. The effective permeability and permittivity can be tuned to negative under specific resonance modes. To further understand the electromagnetic resonance and the physical mechanism of the stopband performance, the dynamically induced field distributions of the switchable all dielectric FSS are calculated using CST Microwave Studio, as shown in Fig. 4. The arrow represents the direction of the field lines and the color indicates the amplitude. From Fig. 4, it can be seen that the electric and magnetic fields are mainly localized in the interior of the dielectric material.

Figure 4(a) shows the electric field distribution of the dielectric ceramics in the plane $x=0 \mathrm{~mm}$ at $39.80 \mathrm{GHz}$. A loop-shaped electric field line distribution appears inside the structure, resulting in a large magnetic field along the $x$-axis, as shown in Fig. 4(c). This type of field distribution inside the structure behaves like a magnetic dipole. Figure 3(b) shows the electric field distribution of the dielectric part in the plane $x=0 \mathrm{~mm}$ at $44.20 \mathrm{GHz}$, which is greatly enhanced relative to the electric field of the incident wave. This configuration of the electric field lines is accompanied by loop-shaped magnetic field lines, as shown in Fig. 3(d). This type of field distribution is equivalent to an electric dipole in the far field. The magnetic dipole and electric dipole in different frequencies lead to the stopband response.

The simulated transmission coefficient of the FSS under normal incidence for TM polarization is shown in Fig. 5(a). Compared with Fig. 3(a), transmitting response emerges at the frequency range of the reflective response for TE polarization. The effective electromagnetic parameters are also retrieved by using the scattering parameters obtained by 


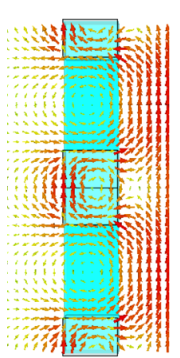

(a)

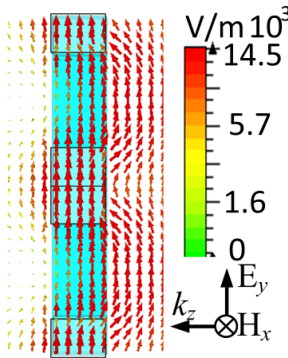

(b)

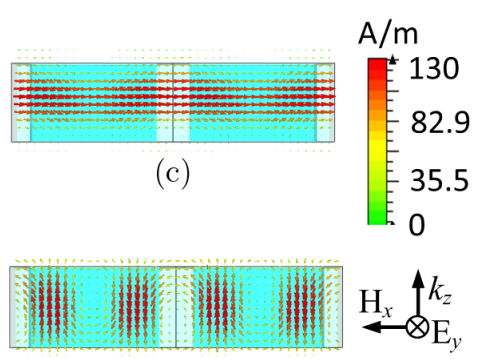

(d)

Fig. 4. Field distributions of the FSS at $39.80 \mathrm{GHz}$ and $44.20 \mathrm{GHz}$ : Electric field distributions for TE polarization at $39.80 \mathrm{GHz}$ (a) and $44.20 \mathrm{GHz}$ (b), magnetic field distributions at $39.80 \mathrm{GHz}$ (c) and $44.20 \mathrm{GHz}$ (d).

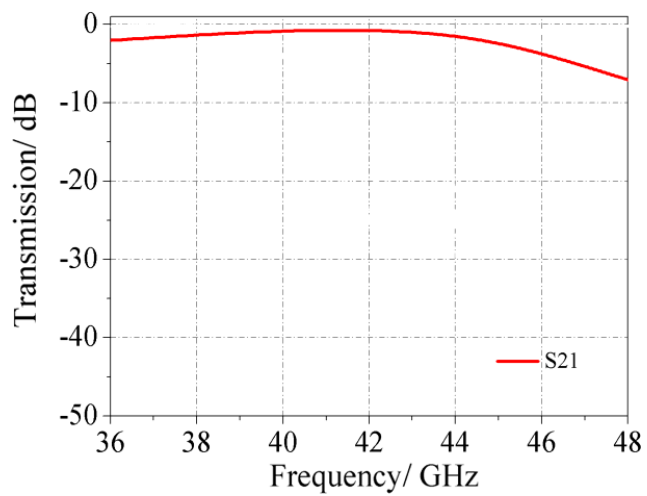

(a)

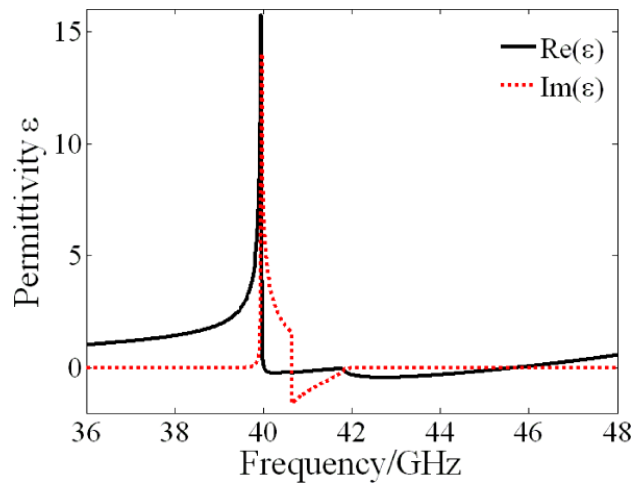

(c)

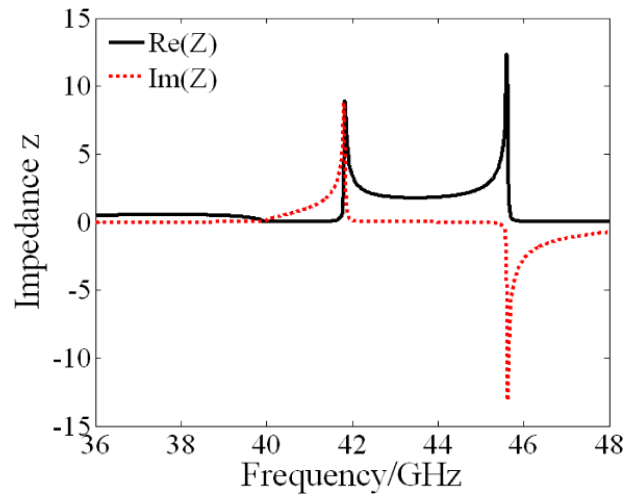

(b)

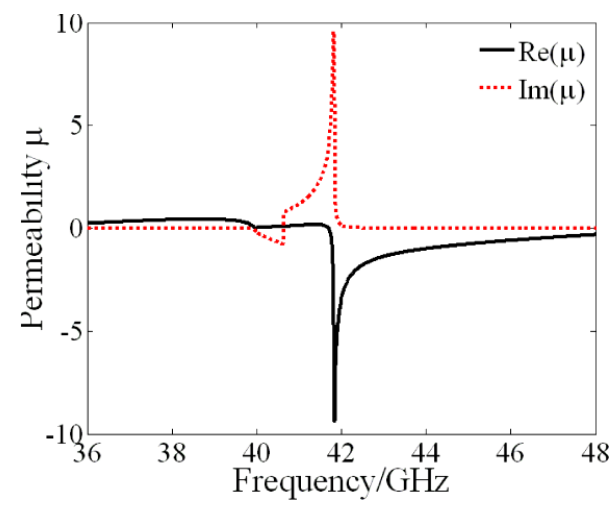

(d)

Fig. 5. Calculated frequency response of the reflect/transmit switchable FSS band under normally incident for TM polarization and the retrieved parameters: (a) transmission coefficient; (b) relative impedance; (c) relative permeability; (d) relative permittivity.

simulation. Figures 5(b)-5(d) show the impedance, retrieved effective permeability and permittivity. Figure 5(b) gives the impedance relative to the free space. It can be seen that the relative impedance of the FSS in frequencies of the passbands approach to 1 , which means the impedance is well-matched to the free space. As shown in Figs. 5(c) and 5(d), the strong electric and magnetic resonances are excited around the frequency where the effective impedance suddenly changes. The permittivity achieves maximum first, and then falls down to the minimum with increasing frequency around $39.8 \mathrm{GHz}$. The characteristic of the permittivity curve is typical of the electric resonance, as shown in Fig. 5(c). And with increasing frequency around $41.9 \mathrm{GHz}$, the permeability also achieves maximum first, and then falls down to the minimum, which displays the magnetic resonance, as shown in Fig. 5(d). The coupling between the electric resonance around $39.8 \mathrm{GHz}$ and the magnetic resonance around $41.9 \mathrm{GHz}$ results in impedance matching, and the left-handed passband is realized.

The dynamic induced field distributions of the FSS are calculated using CST Microwave Studio, as shown in Fig. 6. It can be seen that the electric and magnetic fields are mainly localized in the interior of the dielectric material. 


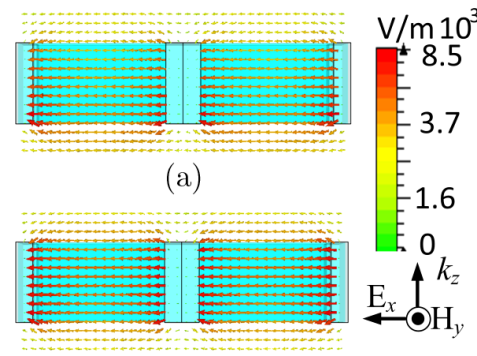

(b)

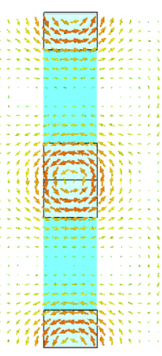

(c)

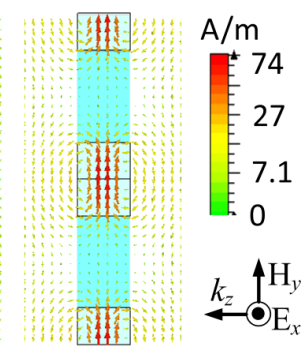

(d)

Fig. 6. Field distributions of the FSS at $39.80 \mathrm{GHz}$ and $41.90 \mathrm{GHz}$ : Electric field distributions for TM polarization at $39.80 \mathrm{GHz}$ (a) and $41.90 \mathrm{GHz}$ (b), magnetic field distributions at $39.80 \mathrm{GHz}$ (c) and $41.90 \mathrm{GHz}$ (d).

Figure 6(a) shows the electric field distribution of the dielectric material in the plane $y=0 \mathrm{~mm}$ at $39.80 \mathrm{GHz}$, which is greatly enhanced relative to the electric field of the incident wave. This configuration of the electric field lines is accompanied by loop-shaped magnetic field lines, as shown in Fig. 6(c). This type of field distribution behaves like an electric dipole. Figure 6(d) shows the magnetic field distribution of the dielectric material in the plane $x=0 \mathrm{~mm}$ at $41.90 \mathrm{GHz}$, which is also greatly enhanced relative to the magnetic field of the incident wave, and the direction of the magnetic field turn to the opposite with the phase changing. This type of field distribution inside the FSS is equivalent to a magnetic dipole in the far field monitor. The coupling between the magnetic dipole and electric dipole in the same frequency leads to the passband.

\section{Conclusions}

In this paper, we present a method of designing reflect/ transmit switchable FSSs based on effective medium theory under quasi-static limit. The FSS, avoiding the use of any metal, is composed purely of all-dielectric metamaterial. With proper design in the frame work of effective medium theory, electric and magnetic resonances can be emerged to achieve mismatched or matched impedances under different polarization. In this way, a bandstop or bandpass response can be achieved. A reflect/transmit switchable FSS composed of square-hole structure is proposed. In addition, the retrieved effective electromagnetic parameters and the dynamically induced field distributions are analyzed to reveal the mechanism of bandstop response.

\section{Acknowledgments}

We gratefully acknowledge the financial support from National Natural Science Foundation of China (Grant Nos. 61331005, 61471388, 11204378, 11274389, 61304393, 61302023), the Natural Science Foundation of Shaanxi Province (Grant No. 2013JM6005) and the Special Funds for Authors of Annual Excellent Doctoral Degree Dissertations of China (Grant No. 201242).

\section{References}

${ }^{1}$ B. A. Munk, Frequency Selective Surfaces: Theory and Design (John Wiley \& Sons, New York, 2000), pp. 1-89.

${ }^{2}$ B. A. Munk, Finite Antenna Arrays and FSS (John Wiley \& Sons, New York, 2000), pp. 12-131.

${ }^{3} \mathrm{~N}$. Behdad, A second-order band-pass frequency selective surface using nonresonant subwavelength periodic structures, Microw. Opt. Technol. Lett. 50(6), 1639 (2008).

${ }^{4}$ F. Yu, S. B. Qu, Z. Xu and J. F. Wang, Investigations on the design of all-dielectric frequency selective surfaces, Proc. Progress in Electromagnetics Research Symp. (2011), pp. 472-475.

${ }^{5}$ G. I. Kiani, A. R. Weily and K. P. Esselle, A novel absorb/transmit FSS for secure indoor wireless networks with reduced multipath fading, IEEE Microw. Wirel. Compon. Lett. 6(16), 378 (2006).

${ }^{6}$ J. A. Bossard, D. H. Werner and T. S. Mayer, A novel design methodology for reconfigurable frequency selective surface using genetic algorithms, IEEE Microw. Antennas Propag. 4(53), 1390 (2005).

${ }^{7}$ T. K. Chang, R. J. Langle and E. A. Parker, An active square loop frequency selective surface, IEEE Microw. Guided Lett. 3(10), 387 (1993).

${ }^{8}$ R. Singh, I. A. I. Al-Naib, M. Koch and W. Zhang, Sharp Fano resonances in $\mathrm{THz}$ metamaterials, Opt. Express 19(7), 6312 (2011).

${ }^{9}$ F. L. Zhang, Q. Zhao, J. Zhou and Sh. X. Wang, Polarization and incidence insensitive dielectric electromagnetically induced transparency metamaterial, Opt. Express, 21(17), 19675 (2013).

${ }^{10}$ J. A. Bossard, X. Liang, L. Li, S. Yun and D. H. Werner, Tunable frequency selective surfaces and negative-zero-positive index metamaterials based on liquid crystals, IEEE Trans. Antennas Propag. 5(56), 1308 (2008).

${ }^{11}$ L. Y. Li, J. Wang, J. F. Wang, H. L. Du, H. Huang, J. Q. Zhang, Sh. B. Qu and Zh. Xu, All-dielectric metamaterial frequency selective surfaces based on high-permittivity ceramic resonators, Appl. Phys. Lett. 106, 212904 (2015).

${ }^{12}$ Y. M. Yang, J. F. Wang, S. Xia, P. Bai, Zh. Li, J. Wang, Zh. Xu and Sh. B. Qu, All-dielectric left-handed metamaterial based on dielectric resonator: Design, simulation and experiment, Chin. Phys. B 20(1), 100864 (2011).

${ }^{13}$ J. Wang, Z. Xu, Z. H. Yu, X. Y. Wei, Y. M. Yang, J. F. Wang and S. B. $\mathrm{Qu}$, Experimental realization of all-dielectric composite cubes/ rods left-handed metamaterial, J. Appl. Phys. 109, 084918 (2011).

${ }^{14}$ J. F. Wang, Sh. B. Qu and Zh. Xu, The design of left-handed metamaterials composed of magnetic resonators and electric resonators, Acta Phys. Sin. 57(8), 5015 (2008). 
${ }^{15}$ B. Du, J. Wang, Zh. Xu, S. Xia, J. F. Wang and Sh. B. Qu, Band split in multiband all-dielectric left-handed metamaterials, J. Appl. Phys. 115, 234104 (2014).

${ }^{16}$ J. H. Barton and C. R. Garcia, All-dielectric frequency selective surface for high power microwaves, IEEE Trans. Antennas Propag. 62(7), 3652 (2014).

${ }^{17}$ J. H. Barton, R. C. Rumpf, R. W. Smith, C. L. Kozikowski and P. A. Zellner, All-dielectric frequency selective surfaces with few number of periods, Prog. Electromagn. Res. B 41, 269 (2012).

${ }^{18}$ V. G. Veselago, The electrodynamics of substances with simultaneously negative values of $\varepsilon$ and $\mu$, Sov. Phys. Uspekhi 10(4), 509 (1968).

${ }^{19}$ D. Kajfez and P. Guillon, Dielectric Resonator, 2nd edn. (Noble Publishing Corporation, Georgia, 1998).
${ }^{20}$ A. Petosa, Dielectric Resonator Antennas Handbook (Artech House, Norwood, MA, 2007), pp. 1-98.

${ }^{21}$ D. R. Smith, J. B. Pendry and M. C. K. Wiltshire, Metamaterials and negative refraction index, Science 305, 788 (2004).

${ }^{22}$ A. Sihvola, Electromagnetic Mixing Formulas and Applications (The Institution of Electrical Engineers, 1999), pp. 3-131.

${ }^{23}$ X. Chen, T. M. Grzegorczyk and B. Wu, Robust method to retrieve the electromagnetic effective parameters of metamaterials, Phys. Rev. E 70, 016608 (2004).

${ }^{24}$ D. Schurig, J. J. Mock and D. R. Smith, Electric-field-coupled resonators for negative permittivity metamaterials, Appl. Phys. Lett. 88, 041109 (2006).

${ }^{25}$ D. M. Pozar, Microwave Engineering, 3rd edn. (John Wiley \& Sons, New York, 2000), p. 132. 